\title{
Experimental and numerical study of omega type bianisotropic metamaterials combined with a negative permittivity medium
}

\author{
Koray Aydin ${ }^{\mathrm{a}, \mathrm{b}, *}$, Zhaofeng $\mathrm{Li}^{\mathrm{a}}$, Serafettin Bilge ${ }^{\mathrm{a}, \mathrm{c}}$, Ekmel Ozbay ${ }^{\mathrm{a}, \mathrm{b}, \mathrm{c}}$ \\ ${ }^{\mathrm{a}}$ Nanotechnology Research Center, Bilkent University, Bilkent 06800, Ankara, Turkey \\ ${ }^{\mathrm{b}}$ Department of Physics, Bilkent University, Bilkent 06800, Ankara, Turkey \\ ${ }^{\mathrm{c}}$ Department of Electrical and Electronics Engineering, Bilkent University, Bilkent 06800, Ankara, Turkey
}

Received 14 July 2007; accepted 30 July 2007

Available online 8 August 2007

\begin{abstract}
We report on the transmission properties of the omega $(\Omega)$ type metamaterials. Transmission through the periodic $\Omega$-only and $\Omega$ wire metamaterials are studied experimentally and numerically. A resonance band gap is observed for the periodic $\Omega$ medium around the resonance frequency of the single $\Omega$ unit cell. A transmission band is observed below the resonance band gap, when the periodic $\Omega$ structure is embedded in a negative permittivity medium composed of thin metallic wire arrays. We also studied the effect of periodicity on the transmission spectra of $\Omega$ type metamaterials.
\end{abstract}

(C) 2007 Published by Elsevier B.V.

PACS : 41.20.Jb; 42.70.-a

Keywords: Metamaterial; Bianisotropic materials; Negative permittivity; Omega media

\section{Introduction}

The responses of materials to the electromagnetic radiation are determined by dielectric permittivity $(\varepsilon)$, magnetic permeability $(\mu)$ and refractive index $(n)$. The materials found in nature usually have positive values of $\varepsilon, \mu$ and $n$. However, by designing artificial structures, one can obtain the desired by values of these material parameters, including the negative ones. The theory of such materials with negative values of permittivity, permeability and refractive index were first discussed by Veselago almost five decades ago [1]. The first experimental results, however, obtained in 2000 by Smith et al., where they demonstrated a metamaterial

\footnotetext{
* Corresponding author. Tel.: +90 312 2901018; fax: +90 3122901018 .

E-mail address: aydin@bilkent.edu.tr (K. Aydin).
}

having simultaneously negative values of $\varepsilon$ and $\mu$ [2] Since then, artificially structured metamaterials have gained burgeoning interest from the scientific community due to their intriguing and exotic electromagnetic properties [3-9]. Composite metamaterials (CMM) are usually designed as a combination of two elements that provide effective-medium response with a permittivity of $\varepsilon(\omega)<0$ and permeability of $\mu(\omega)<0$ simultaneously over a certain frequency range, so that the resulting index of the refraction of the effective medium becomes $n_{\mathrm{eff}}=\sqrt{\varepsilon} \sqrt{\mu}<0$. Split-ring resonators (SRR) are widely used to obtain an effective negative permeability at frequencies close to the magnetic resonance frequency of SRR [10]. By combining SRRs with a negative permittivity medium composed of periodic thin wire media [11], one can obtain a lefthanded transmission band [3].

In a recent paper by Tretyakov et al., a backward wave media due to the bianisotropic characteristic of 
$\Omega$ resonators are reported [12]. The authors proposed an alternative metamaterial composed of periodic arrangement of periodic omega patterns and thin wire media. Omega structures were proposed by Saadoun and Engheta back in 1992 [13]. These types of structures are composite electromagnetic materials with a proper combination of $\Omega$-shaped metallic inclusions in a host dielectric medium. The omega structure resembles the Greek letter omega, and consists of a C-shaped ring resonator with two wires connected to both ends. These metamaterials have bianisotropic characteristics and also named as pseudochiral media [13]. Electric and magnetic polarizations are induced by both electric and magnetic fields in bianisotropic media.

\section{Resonance frequency of single omega unit cell}

The resonance of omega structures can be investigated by studying the frequency response of single omega unit cells. Previously, Aydin et al. reported the measured and simulated resonance characteristics of split-ring resonator unit cell [14]. Here, we follow the same methodology to understand the resonance of omega structure.

A dip in the transmission spectrum of $\Omega$ unit cells can be attributed to the resonant nature of these structures. We performed transmission measurements on the omega unit cell that is shown in Fig. 1(a). The parameters as given in the figure are $R=1.19 \mathrm{~mm}$, $L=1.8 \mathrm{~mm}$ and $w=0.45 \mathrm{~mm}$. Omega patterns are obtained by etching the copper that is deposited on a FR4 substrate. The thickness of the copper and FR4 are $30 \mu \mathrm{m}$ and $1.6 \mathrm{~mm}$. The FR4 substrate used in these experiments have a dielectric constant, $\varepsilon_{\mathrm{s}}=4.3$ and loss tangent, $\delta \sim 0.025$.

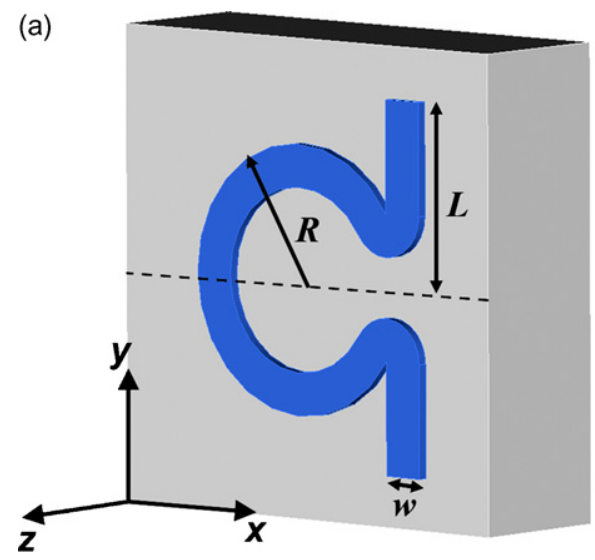

In the measurements, we used monopole antennae as a source and receiver. The antennae are connected to the HP-8510C network analyzer to measure the $S$ parameters. We also performed numerical simulations to check the validity of the experimental results. Simulations were performed by using the commercial software, CST Microwave Studio, which is a 3D full-wave solver that employs the finite integration technique. A single layer of the $\Omega$ structure along the propagation direction is subjected to an incident plane wave. Open boundary conditions are employed along the propagation direction. The structure is assumed to be periodic and infinite along the directions that are perpendicular to the propagation direction. The transmission amplitudes are obtained by probing the fields at a distant point from the structure.

We measured single $\Omega$ unit cell for two different orientations. In the first orientation, the incident field propagates along the $x$ direction, with $E$ and $H$ along the $y$ and $z$ directions, respectively (see Fig. 1(a) for the directions). For this case, $E$ is parallel to the tails of the omega. The measured and simulated S21 spectra for $\Omega$ unit cell are shown with black line and blue dasheddotted line in Fig. 2. The transmission dip is appeared at $10.75 \mathrm{GHz}$ in the measurements and simulations. In previous work, we developed a method to check the resonance of split-ring resonators, in which we closed the splits of the SRR structure [4]. Here, we follow a similar method and close the gap region of the $\Omega$ structure by connecting the tails as shown in Fig. 1(b). The resulting structure is named as a closed $\Omega$ for the convenience. The measured frequency response of closed $\Omega$ structure is shown with gray dashed line in Fig. 2(a). As clearly seen, the closed $\Omega$ unit cell does not show any resonant behavior since the incident EM wave is totally transmitted.

(b)

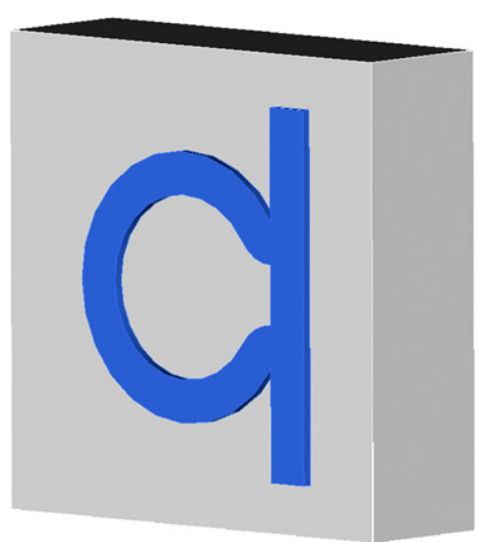

Fig. 1. Schematic drawings of (a) single omega unit cell and (b) closed omega unit cell. 


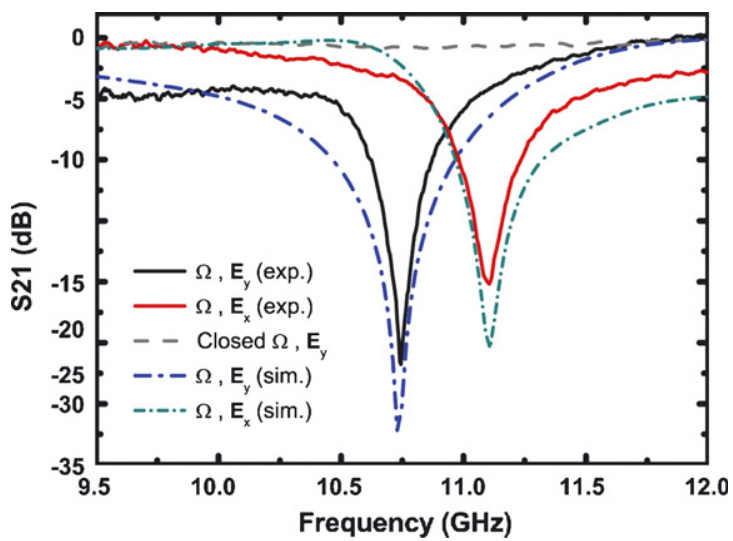

Fig. 2. Measured frequency responses of omega unit cells with incident $E$ field along $y$-axis (black line) and $x$-axis (red line). The simulation results are plotted with blue and green dashed-dotted lines. Gray dashed line is the measured data for close omega unit cell where $E$ is along $y$-axis.

We then changed the orientation of the $\Omega$ structure, where the field propagates along $y$-direction and $E$ is along the $x$-direction. The magnetic field is not changed and is still along the $z$-direction. To visualize better, the tails are perpendicular to the $E$ field in this second case. The resonance frequency of rotated $\Omega$ unit cell was observed at a higher frequency, $11.1 \mathrm{GHz}$, as observed in the measurements (red line) and simulations (green dashed-dotted line). The $\Omega$ structure with tails parallel to the $E$ field has a lower resonance frequency compared to the $\Omega$ structure with tails perpendicular to the $E$ field. When the tails are parallel to the $E$, the dipole like part of $\Omega$ (the cut wires) face additional electric resonance and this in turn results in a reduction of resonance frequency. Increasing the tail length of the $\Omega$ structure decreases the resonance frequency furthermore [15].

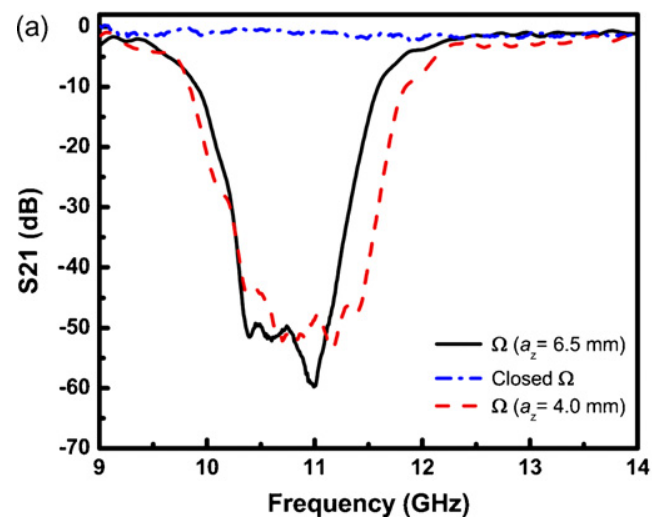

\section{Tranmission characteristics of omega type metamaterials}

In the previous section, we observed resonance for the unit cell of $\Omega$ structure. It is well known that if resonant structures are arranged periodically, a band gap occurs around the resonance frequency due to the strong coupling between the resonant structures. Such an effect was previously observed for SRR structures $[2,3]$. We arranged $\Omega$ type resonators on the FR4 substrate with periodicities $a_{x}=a_{y}=5 \mathrm{~mm}$. We initially chose the periodicity between the omega boards (i.e. along the $z-$ direction) as $a_{z}=6.5 \mathrm{~mm}$. The number of unit cells in the $x, y$, and $z$ directions are 5,30, and 32. Standard high gain microwave horn antennae are used as transmitters and receivers which are connected to HP 8510-C network analyzer.

Fig. 3(a) shows the measured transmission spectra periodic $\Omega$ media. Periodic $\Omega$ media with $a_{z}=6.5 \mathrm{~mm}$ has a band gap at frequencies $10.10-11.50 \mathrm{GHz}$ (black line). The transmission spectrum of periodic closed $\Omega$ structure is flat throughout the frequency range of interest, meaning that the band gap is due to the magnetic resonance of the $\Omega$ structure and electric resonance due to the dipole-like structures. The resonance frequency of $\Omega$ unit cell was observed at $10.75 \mathrm{GHz}$. The band gap is formed around this resonance frequency due to the coupling in all directions. We then reduced the periodicity, in order to investigate the effect of coupling on the width of the resonance band gap. The red dashed line in Fig. 3(a) plots the transmission spectrum of periodic $\Omega$ media with a periodicity of $a_{z}=4.0 \mathrm{~mm}$. The band gap for the $\Omega$ array with reduced periodicity is observed between 10.00 and $11.75 \mathrm{GHz}$. Decreasing the periodicity between the omega boards results in an increase in

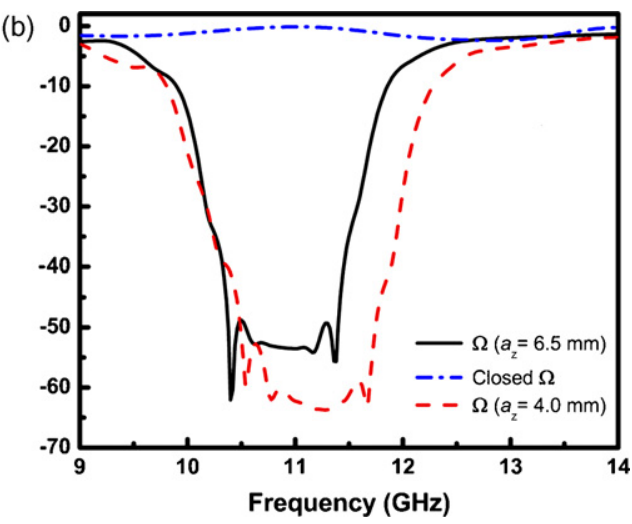

Fig. 3. (a) Measured and (b) simulated transmission spectra of periodic omega (black line) and closed omega structures (blue dashed-dotted line) with a periodicity of $a_{z}=6.5 \mathrm{~mm}$. Red dashed line plots the measured transmission spectrum of periodic omega media for $a_{\mathrm{z}}=4.0 \mathrm{~mm}$. 
the band width of the resonance band gap. Since the neighboring $\Omega$ boards are closer for $a_{z}=4.0 \mathrm{~mm}$, the coupling increases, which results in a $0.35 \mathrm{GHz}$ increase in the width of the band gap. The simulation results are plotted in Fig. 3(b) and there is a good agreement. In the simulations, the band gaps appeared for periodic $\Omega$ with $a_{z}=6.5 \mathrm{~mm}$ at $10.10-11.70 \mathrm{GHz}$ and for $a_{z}=4.0 \mathrm{~mm}$ at $10.00-12.05 \mathrm{GHz}$. The lower edges of the band gap are consistent with the measured results, however the upper edge appears at higher frequencies. We believe that this may be due to the misalignment of the boards in the measurements or fabrication errors resulting in a deviation from the structures' parameters.

The $\Omega$ structure has bianisotropic properties meaning that the dielectric permittivity and magnetic permeability of the effective $\Omega$ medium depends both on the electric and magnetic fields. The magnetic and electric resonances are coupled in the periodic $\Omega$ structure. The presence of electric resonance affects the strength of the magnetic response. Therefore, by using $\Omega$ media, one may not have negative permeability values inside the band gap. To make a comparison, SRR structures are composed of high capacitive elements, splits, and gaps that enhance the magnetic response. However, the contribution of magnetic resonance in $\Omega$ media is not as strong as in the case of SRR structures. Tretyakov et al. performed numerical analysis where the retrieved effective permeability of periodic $\Omega$ media does not possess negative values even though it exhibits resonance behavior due to a magnetic response [12]. On the other hand, the $\Omega$ structure is still interesting, because a backward wave exists if the sum $\operatorname{Re}(\varepsilon+\mu)$ is negative at certain frequencies although both parameters are not negative. The wave vector inside a backward media is against the wave vector of the incident EM radiation. The authors arranged periodic $\Omega$ media with a negative permittivity medium composed of thin metallic wires in order to verify the backward wave media.

We composed a metamaterial combined of $\Omega$ resonators and thin wire arrays. It is well known that, the negative permittivity at microwave frequencies can be achieved by proper arrangement of thin metallic wire medium. The dielectric permittivity takes negative values below the plasma frequency of the wire medium. For this purpose, we have constructed a wire board periodic along the $x$ direction with a periodicity of $a_{x}=5 \mathrm{~mm}$. The width and height of thin wire are $w=0.45 \mathrm{~mm}$ and $h=5 \mathrm{~mm}$. Along the $y$-direction, the wires are continuous and the total length of the wires is $150 \mathrm{~mm}$. When the wire boards are arranged along $z$-direction with a periodicity of $a_{z}=6.5 \mathrm{~mm}$, the plasma frequency of the wire is observed at 13.00 GHz. The transmission spectrum of the wire media is shown in Fig. 4(a) with a blue dashed line. The simulations predict the plasma frequency around 13.10 GHz as given in Fig. 4(b).

We also fabricated a composite metamaterial (CMM) board, in which the front side of the board has the $\Omega$ patterns and the back side of the board has the wire arrays. The center of the wire coincides with the center of the ring part of the $\Omega$ patterns. The measured and simulated results for the CMM is given in Fig. 4(a and b), respectively. The black lines plot the transmission spectra of periodic omega-only media. The transmission spectra of the resulting composite metamaterial are shown by the red lines in the figure. A transmission band is observed below the magnetic resonance band gap of periodic $\Omega$ medium. The
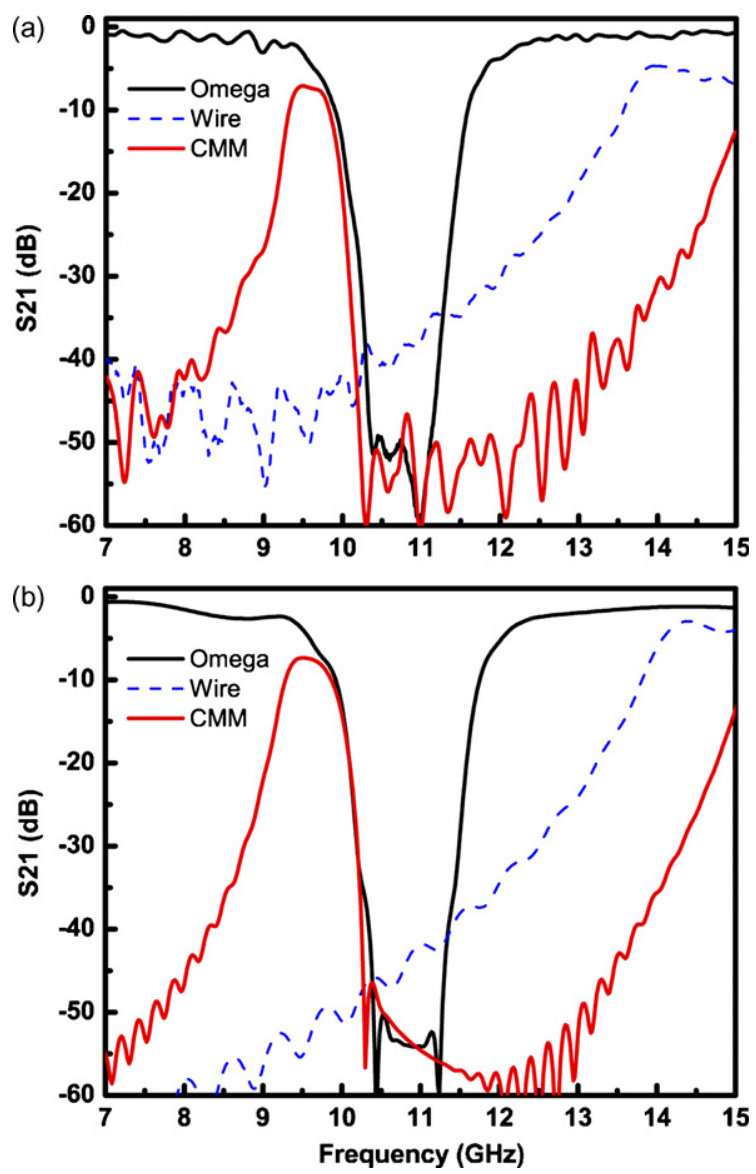

Fig. 4. (a) Measured and (b) simulated transmission spectra of periodic omega (black line) and wire (blue dashed line) media with a periodicity of $a_{\mathrm{z}}=6.5 \mathrm{~mm}$. Transmission spectrum of composite metamaterial where omega and wire structures are arranged periodically is plotted with red line. 


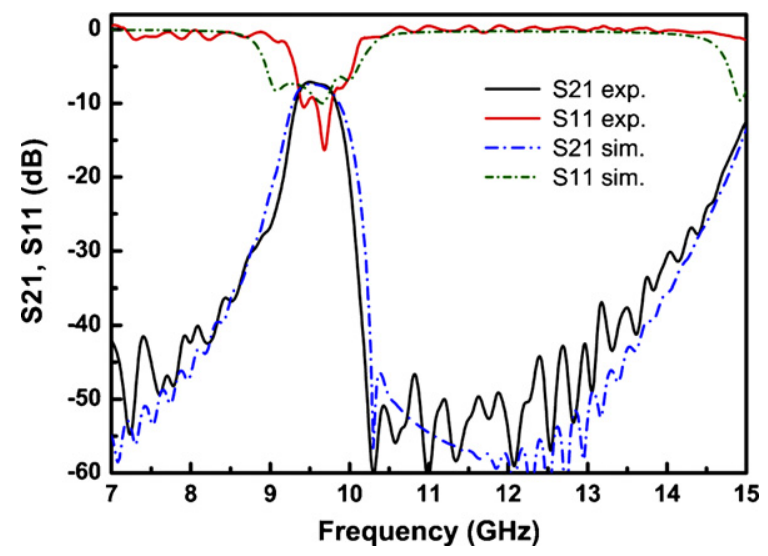

Fig. 5. The transmission and reflection spectra of CMM for $a_{\mathrm{z}}=6.5 \mathrm{~mm}$

measured transmission band of CMM is between 9.15 and $10.05 \mathrm{GHz}$. The maximum transmission is around $-7.0 \mathrm{~dB}$. In the simulations a wider band is calculated to be at $9.05-10.12 \mathrm{GHz}$ and the transmission peak is around $-7.3 \mathrm{~dB}$. The upper edge of CMM pass band coincides with the lower edge of the periodic $\Omega$ band gap. We also measured and simulated the reflection spectrum for the CMM. $S 11$ and $S 21$ parameters of the CMM structure are given in Fig. 5. As seen in the figure, the transmission spectra obtained from the measurements and simulations are in good agreement. The measurement results show that the average reflection within the transmission band of CMM is around $-10 \mathrm{~dB}$. The minimum reflection is measured as $-16.3 \mathrm{~dB}$ at $9.68 \mathrm{GHz}$. The reflection band is wider in the simulated reflection spectrum, where the average reflection is around $-8 \mathrm{~dB}$. In the simulations, the minimum reflection appeared at $9.66 \mathrm{GHz}$ with a value of $-10 \mathrm{~dB}$. Aydin et al. reported low reflection $(-38 \mathrm{~dB})$ from the surface of left-handed metamaterial composed of SRR and wire arrays [9]. The matching of SRR-wire metamaterial is better than the omega-wire metamaterial. This may be due to the value of negative permeability of the resonant structures. The SRR structure provides higher negative values compared to that of omega. However, better impedance matching can be achieved by a meticulous design of omega-wire metamaterial [12].

We also performed transmission measurements and simulations for the periodicity $a_{z}=4.0 \mathrm{~mm}$. The results are provided in Fig. 6(a and b). The previous is the measurement results; the latter is the simulation results. The simulated plasma frequency appeared at $16.4 \mathrm{GHz}$ for wire array that is stacked with a periodicity of $a_{z}=4.0 \mathrm{~mm}$ (data not shown). As expected, reducing the periodicity between the neighboring wire boards
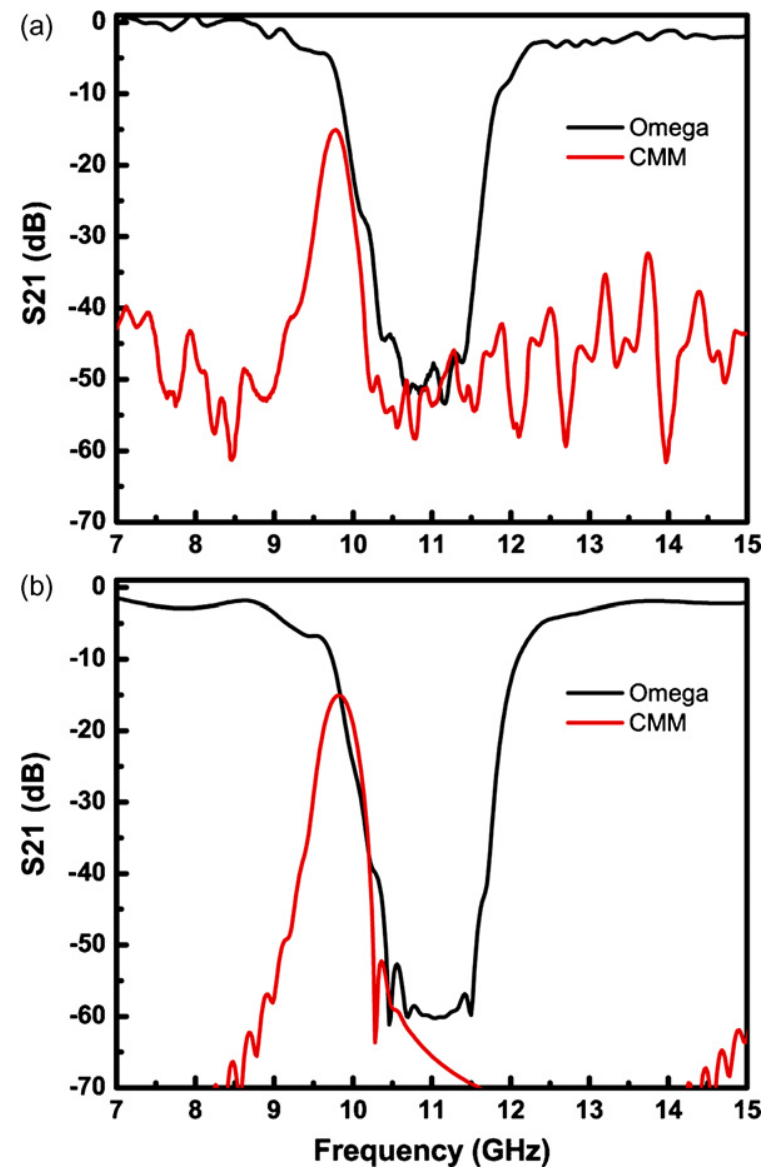

Fig. 6. (a) Measured and (b) simulated transmission spectra of periodic omega structure (black line) and CMM (red line) with a periodicity of $a_{\mathrm{z}}=4.0 \mathrm{~mm}$.

resulted in an increase of the plasma frequency. The black lines in the figure plot the transmission spectra of only omega media and the red lines correspond to the transmission spectra of composite metamaterial (omega-wire media). The transmission band is observed between 9.55 and $10.00 \mathrm{GHz}$ in the measurements and $9.55-10.05 \mathrm{GHz}$ in the simulations. The maximum transmission is $-15 \mathrm{~dB}$ both in the measurements and simulations, meaning that the agreement is good. The lower edge of the resonance band gap of omega media coincides with the upper edge of the transmission band as in the case of metamaterial with higher periodicity. However, the maximum value of transmission is reduced for when the periodicity is decreased. Since the plasma frequency appears at higher frequencies, the effective dielectric permittivity of the CMM possesses higher negative values at the transmission band which makes it difficult to obtain a good matching.

The existence of a pass band for the composite metamaterial within the respective stop bands of SRR- 
only and wire-only media is intuitively considered as evidence for left-handed behavior [2,3]. However, a transmission band within the negative permittivity and permeability region may not exhibit left-handed characteristics. Recently, $\pi$-shaped metamaterials have been reported to exhibit a transmission band with right-handed characteristics [16]. The metamaterials composed of $\pi$-shaped structures are also of the bianisotropic type. The transmission band in Ref. [12] is observed at the lower edge of the resonance band gap of $\pi$-shaped resonator, similar to the experimental results that we have obtained. In our case, we observed a transmission band below the resonance frequency, which means that within the transmission band the omega metamaterial exhibits a right-handed behavior [16]. However, a right-handed transmission band may result in a backward wave regime [12]. It is interesting to observe a pass band within negative permittivity region of thin wire media, and this effect needs to be investigated in detail. The metamaterial composed of $\Omega$ structures have bianisotropic characteristics. Following the analysis of Tretyakov et al. in Ref. [12], we believe that the observed transmission band may correspond to the backward wave regime, although the effective permeability and refractive index may not be negative.

\section{Conclusion}

We have studied the transmission resonance of the $\Omega$ unit cells with different orientations with respect to the incident electric field. The transmission characteristics of periodic omega structures are reported. The band gap is due to the coupled electric and magnetic resonances since the $\Omega$ type structures have bianisotropic properties. A transmission band is observed when the periodic omega media is brought together with the periodic wire array. The lower edge of the resonance band gap of omega media coincides with the upper edge of the transmission band of the CMM, meaning that the transmission band may not exhibit left-handed characteristics. This transmission band, however, could result in backward wave propagation in the metamater- ial. We believe that the metamaterials composed of bianisotropic structures could be an alternative type of metamaterials, and novel properties could be obtained with detailed analyses.

\section{Acknowledgments}

This work is supported by the European Union under the projects EU-NoE-METAMORPHOSE, EU-NoEPHOREMOST, and TUBITAK under Projects Nos. 104E090, 105E066, 105A005, and 106A017. One of the authors (E.O.) also acknowledges partial support from the Turkish Academy of Sciences.

\section{References}

[1] V.G. Veselago, Engl. Trans. Sov. Phys. Ups. 10 (1968) 509.

[2] D.R. Smith, W.J. Padilla, D.C. Vier, S.C. Nemat-Nasser, S. Schultz, Phys. Rev. Lett. 84 (2000) 4184.

[3] K. Aydin, K. Guven, M. Kafesaki, L. Zhang, C.M. Soukoulis, E. Ozbay, Opt. Lett. 29 (2004) 2623.

[4] N. Katsarakis, Th. Koschny, M. Kafesaki, E.N. Economou, C.M. Soukoulis, Appl. Phys. Lett. 84 (2004) 2943.

[5] K. Aydin, K. Guven, C.M. Soukoulis, E. Ozbay, Appl. Phys. Lett. 86 (2005) 124102.

[6] K. Aydin, I. Bulu, E. Ozbay, Opt. Express 13 (2005) 8753.

[7] R.S. Penciu, M. Kafesaki, T.F. Gundogdu, E.N. Economou, C.M. Soukoulis, Photon. Nanostruct.: Fundam. Appl. 4 (2006) 12.

[8] K. Guven, K. Aydin, E. Ozbay, Photon. Nanostruct.: Fundam. Appl. 3 (2005) 75.

[9] K. Aydin, I. Bulu, E. Ozbay, Appl. Phys. Lett. 90 (2007) 254102.

[10] J.B. Pendry, A.J. Holden, D.J. Robbins, W.J. Stewart, I.E.E.E. Trans, Microw. Theory Tech. 47 (1999) 2057.

[11] J.B. Pendry, A.J. Holden, W.J. Stewart, I. Youngs, J. Phys. Condens. Matter. 10 (1998) 4785.

[12] S.A. Tretyakov, C.R. Simovski, M. Hudlicka, Phys. Rev. B 75 (2007) 153104

[13] M.M.I. Saadoun, N. Engheta, Microw. Opt. Tech. Lett. 5 (1992) 184.

[14] K. Aydin, I. Bulu, K. Guven, M. Kafesaki, C.M. Soukoulis, E. Ozbay, New J. Phys. 7 (2005) 168.

[15] K. Aydin, Z. Li, M. Hudlicka, S.A. Tretyakov, E. Ozbay, New J. Phys. 9 (2007) 326.

[16] Z.-G. Dong, S.Y. Lei, M.X. Xu, H. Liu, T. Li, F.M. Wang, S.N. Zhu, Phys. Rev. B 75 (2007) 075117. 